\title{
Determination of effective antecedent rainfall for debris flow forecast based on soil moisture content observation in Jiangjia Gully, China
}

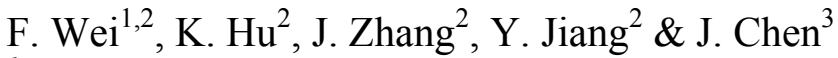 \\ ${ }^{1}$ Key Laboratory of Mountain Hazards and Surface Process, \\ Chinese Academy of Science, Chengdu, China \\ ${ }^{2}$ Institute of Mountain Hazards and Environment, \\ Chinese Academy of Sciences, Chengdu, China \\ ${ }^{3}$ School of Geographical Sciences, Southwest University, Chongqing, \\ China
}

\begin{abstract}
Effective antecedent rainfall (EAR), one of important factors for debris flow forecast, has different ways of influencing debris flow initiation for different types of debris flows. For soil-mechanics-typed debris flow, EAR impacts its initiation by changing the soil moisture content, and for hydromechanics-typed debris flow, EAR impacts its initiation by increasing the surface runoff. For this reason, the methods of determining EAR for different types of debris flows are different. The method for the former type of debris flow is discussed in this paper. Under the assumptions that soil moisture content due to antecedent rainfall decays in the same way as EAR, and that the decays for different events are mutually independent, the variation of EAR can be carried out through analyzing the variation of soil moisture content. The EAR for debris flow forecast can then be determined. The decaying of soil moisture content had been analyzed according to the field observation of rainfall and soil moisture content in Jiangjia Gully in Yunan Province, where debris flows are caused by soil mechanical changing triggered by rainfall. Based on the field observation and data analysis, the formula of calculating EAR for Jiangjia Gully is made out with least-squares procedure.
\end{abstract}

Keywords: debris flow, forecast, antecedent rainfall, effective rainfall, soil moisture content. 


\section{Introduction}

Rainfall is an important factor of rainfall-typed debris flow forecast because it is the direct triggering factor of debris flow initiation. In a debris flow event, the rainfall impacting its initiation includes antecedent rainfall and current rainfall. The former consists of effective antecedent rainfall (EAR) which influences the event and loss of rainfall which is lost through surface runoff, groundwater runoff and evaporation etc. The latter consists of effective current rainfall (ECR) which is the part of rainfall before the event and surplus rainfall which is the part of rainfall after the event. Both EAR and ECR are the important factors of debris flow forecast because they impact debris flow initiation jointly. ECR can be obtained through rainfall forecast and rainfall monitoring, but it's difficult to calculate or evaluate EAR, and there is no reliable approach at present. In past researches, Senoo et al (1985) gave an equation to calculate EAR as $R_{a}=\sum_{t=1}^{14} a_{t} R_{t}$, where $R_{a}$ is EAR, $R_{t}$ is the daily-rainfall on the $t$-day before, $a_{t}=0.5^{t / T}$ is decrement rate, where $T$ is the half life of rainfall. Rainfall in the last 14 days is considered in this equation [1]. This equation was used widely in Japan and was modified in later applications (such as Fujii et al. [3]; Kubota et al. [2]; Hayashi et al. [4] and Senoo et al. [5]), but the form of the equation was not changed [2-6]. Tan [6] adopted monthly rainfall as antecedent rainfall in the research on the character of rainfall vertical distribution and critical rainfall of debris flow forecast in Babuli Gully, Sichuan Province, China and employed the equation of Senoo (1985) in other research (1989) [7-8]. The equation $P_{a 0}=P_{1} K+P_{2} K^{2}+P_{3} K^{3}+\ldots+P_{n} K^{n}$ was used by Chen [9] to calculate EAR in debris flow forecast, where $P_{a 0}$ is EAR, $P_{1}, P_{2}, P_{3}, P_{n}$ are the rainfall respectively on the $1^{\text {st }}, 2^{\text {nd }}, \mathrm{n}^{\text {th }}$ day past, $K$ is the decrease coefficient. In this equation, $K$ is suggested from 0.8 to 0.9 , and $n=20$. Tan et al. [8] considered the action of antecedent rainfall in his research of debris flow forecast for railway. But no equation was given out, only a coefficient $K$ $(\geq 1)$ was used to correct the rainfall. Fan el at [10] used a recession coefficient $\alpha$ to express EAR in the research on critical threshold rainfall of debris flow occurrence in central Taiwan and adopted Fedora's (1989) recession coefficient of antecedent precipitation $k=0.881+0.00793 \times \ln (a)$ in the research of storm runoff to determine $\alpha=\sqrt{k}$, where $\alpha$ is the catchment area [11,12]. According to these researches, two parameters are difficult to determine in calculating EAR: the decay coefficient and the number of days. Furthermore, all rainfalls that occurred in a certain period have been considered, although some light rainfalls may be lost completely. It seems just for the reason that the EAR is difficult to determine or evaluate accurately, the EAR has been not considered in many researches of debris flow forecast. However, it's a very important factor impacting debris flow initiation, it can't be neglected in debris flow forecast. In order to resolve this problem, the decay process of EAR has to be researched.

According to the field observation and the research of $\mathrm{Wu}[13]$, the initiation of debris flow can be classified into two types. One is soil-mechanics-typed that the soil on the slope becomes unstable and forms debris flow while rainfall increases the soil moisture content and changes the characteristics of soil 
mechanics. And the other one is hydromechanics-typed that debris on slope or valley beds is eroded by increasing runoff and then forms debris flow. For the former, EAR should be researched through analyzing the decaying of soil moisture content after rainfall, and for the latter, it should be researched through analyzing the decaying of runoff after rainfall. The decaying of runoff has been researched largely in hydrology, and some results of them can be used in determining EAR for hydromechanics-typed debris flow forecast. The method of determining EAR discussed in this paper is for the soil-mechanics-typed debris flow forecast. It is researched through observing the rainfall and soil moisture content in Jiangjia Gully in the province of Yunnan, China (Fig. 1), where debris flows are caused by soil mechanics changing triggered by rainfall, and where Dongchuan Debris flow Observation Station, Chinese Academy of Sciences is located.

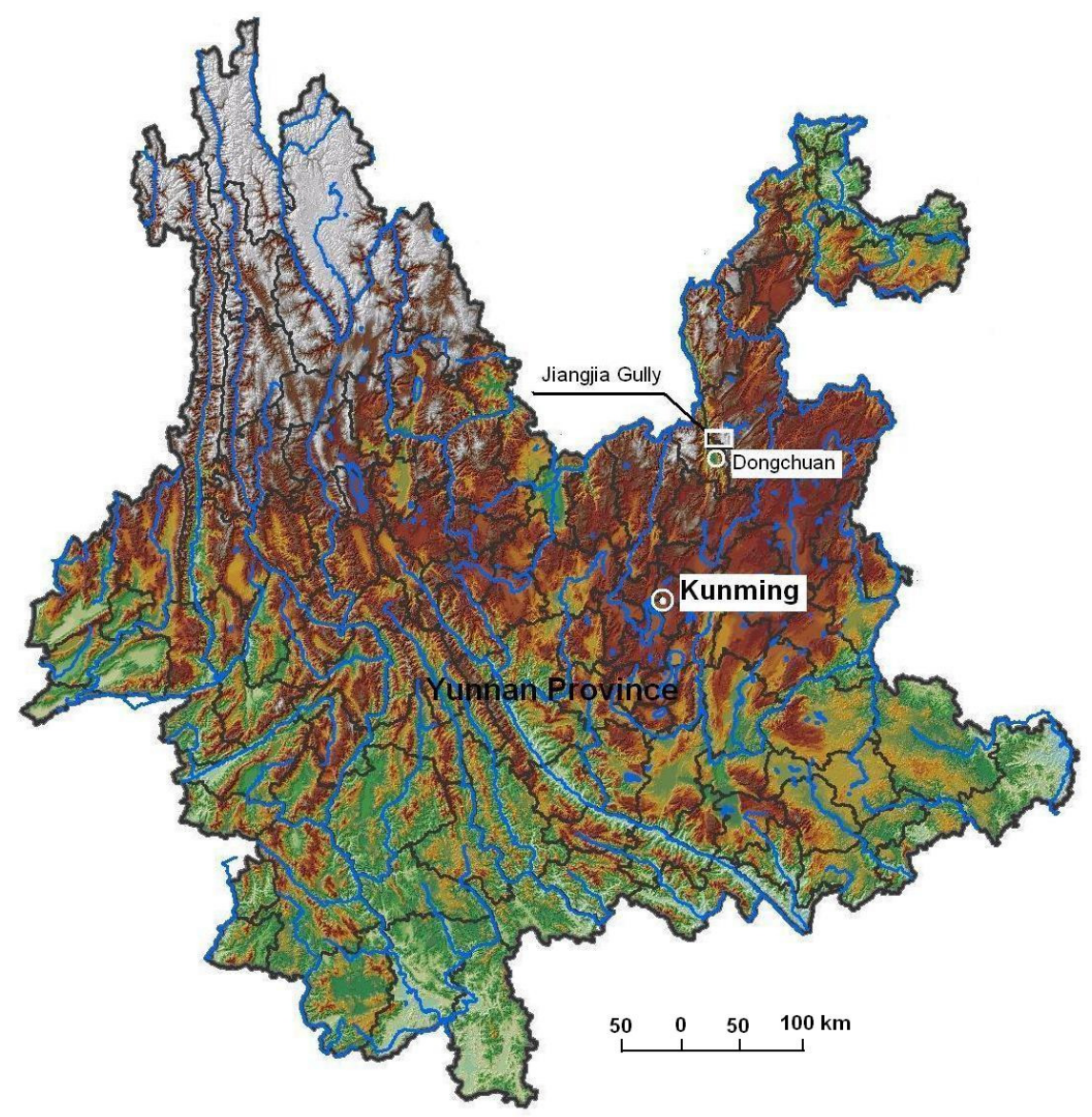

Figure 1: The position of Jiangjia Gully. 


\section{Relation between EAR and variation of soil moisture content}

For soil-mechanics-typed debris flow, soil moisture content is crucial for debris flow initiation, and the increase of soil moisture content is caused by rainfall. So the soil moisture content and rainfall are closely related. The soil moisture content increases rapidly during rainfall but decreases rapidly after the rainfall for runoff and evaporation, etc. The rapid decrease of soil moisture content causes its action on debris flow initiation to weaken fast. Because the soil moisture content is caused by rainfall, it means the impact of antecedent rainfall on debris flow initiation weakens rapidly. In other words, its EAR decreases rapidly. Therefore it can be assumed that the decrease of EAR coincides with the decrease of soil moisture content and decays in the same manner. Under this assumption, decaying of EAR can be determined through analyzing the decaying of soil moisture content.

Of course, more rainfall events may happen while the effective rainfall of the former event doesn't lose completely. It will induce superimposing of EAR from different rainfall events, as does the soil moisture content. In order to make it simple, it can be assumed that the decrease processes of EAR of different events are mutually independent, and that the decrease processes of soil moisture contents due to different rainfalls events are also mutually independent. Under this assumption, the total antecedent effective rainfall is the linear superimposing of the effective rainfall of every rainfall event, so dose the total soil moisture content.

$$
\begin{gathered}
E F=E F_{1}+E F_{2}+\ldots+E F_{n} \\
E W=E W_{1}+E W_{2}+\ldots+E W_{n}+c
\end{gathered}
$$

Where $E F$ and $E W$ are total EAR and total soil moisture content, $E F_{n}$ and $E W_{n}$ are the effective rainfall and soil moisture content of the rainfall event on the $n$ day before, $\mathrm{c}$ is a constant.

Generally soil moisture content increases along with the increase of rainfall. However, it is not a strict increase because same precipitations with different intensities and processes may cause different infiltrations and induce different soil moisture contents. In order to simplify it, it is assumed that in the 24-hour statistics period that soil moisture content increases linearly with the increase of rainfall. Furthermore, the increase of soil moisture content is zero while the rainfall is zero. So the relation between EAR and the soil moisture content is reduced to a simple coefficient relation. Set the effective rainfall of the rainfall event on the i-day before $E F_{i}=f(F, i)$, where $\mathrm{F}$ is the rainfall on the $i$-day before, and the soil moisture content due to the rainfall event on the $i$-day before $E W_{i}=f(W, i)$, where $\mathrm{W}$ is the increase of soil moisture content due to the rainfall on the i-day before, then $f(F, i)$ and $g(W, i)$ should have the same functional form based on the assumption that the decaying of soil moisture content and effective rainfall follow the same manner of decaying. Thus, the difficult problem of determining antecedent effective rainfall is transformed to the problem of determining soil moisture content, which can be resolved by field observation and statistics analysis. 


\section{Observation of rainfall and soil moisture content}

In order to determine the decay process of soil moisture content after rainfall and the functional form of $g(W, i)$, the rainfall and soil moisture content were observed for 39 days continuously in field in the rainy season of 2003.

The period of observation is from 30 July to 6 September. The position of observation is in Jiangjia Gully, Yunnan Province, China. Most of the debris flows in this catchment are soil-mechanics-typed. The debris flows events appear about 15 times per year averagely triggered by rainfall. There are good observation conditions here because the Dongchuan Debris Flow Observation Station, Chinese Academy of Sciences is located in this catchment. Three points with different characters were selected to observe the rainfall and soil moisture content (Fig. 2). The first point is on a semi-naked slope with $31^{\circ}$ gradient and

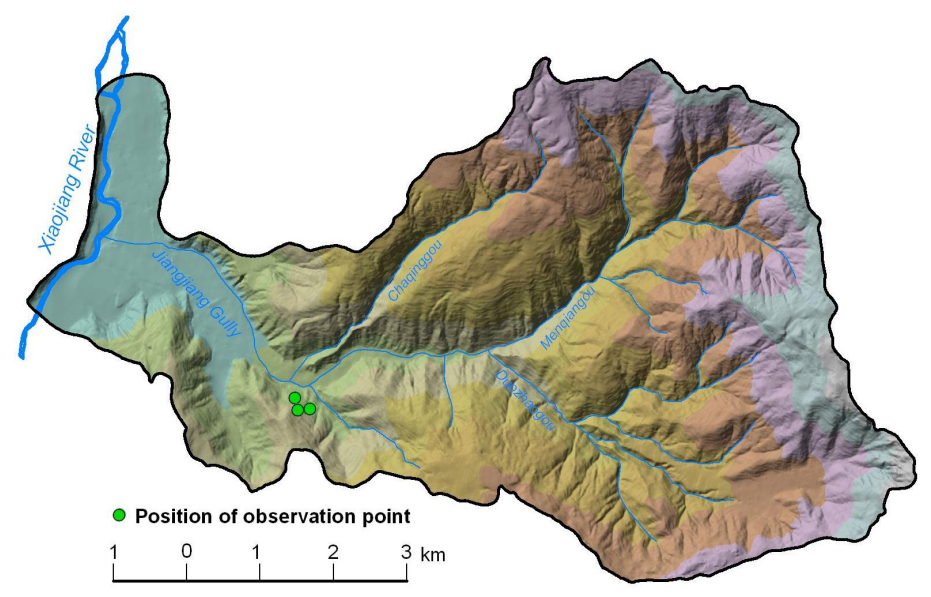

Figure 2: The catchment of Jiangjia Gully and position of the observation points.

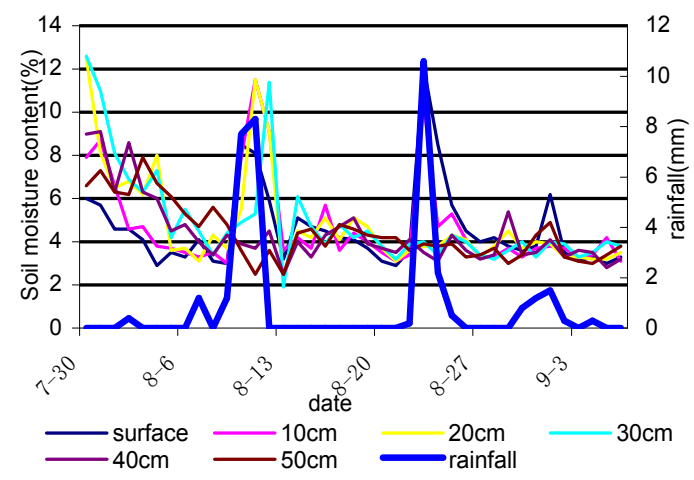

Figure 3: Observation data in the first observation point. 
$\mathrm{NE} 25^{\circ}$ aspect. The second point is on a forest slope with $40^{\circ}$ gradient and NE20 $0^{\circ}$ The third point is on naked flat ground. A profile with $50 \mathrm{~cm}$ depth was made to observe the soil moisture content in different depth (surface, $10 \mathrm{~cm}, 20 \mathrm{~cm}, 30 \mathrm{~cm}$, $40 \mathrm{~cm}$ and $50 \mathrm{~cm}$ ) in every observation point.

The observation data of rainfall and soil moisture content on 3 observation points are illustrated in Fig. 3 to Fig. 5.

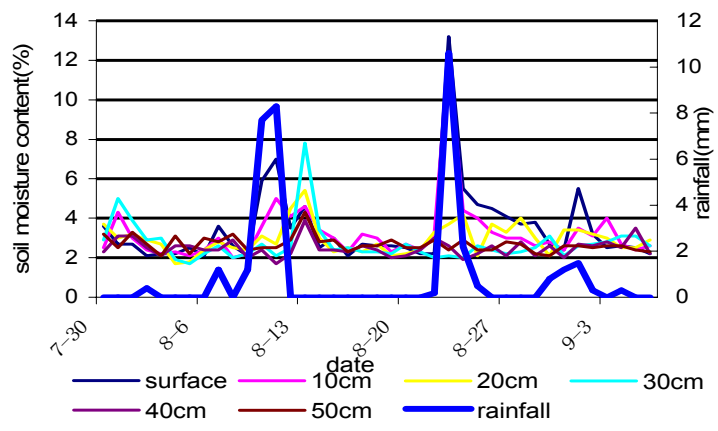

Figure 4: Observation data in the second observation point.

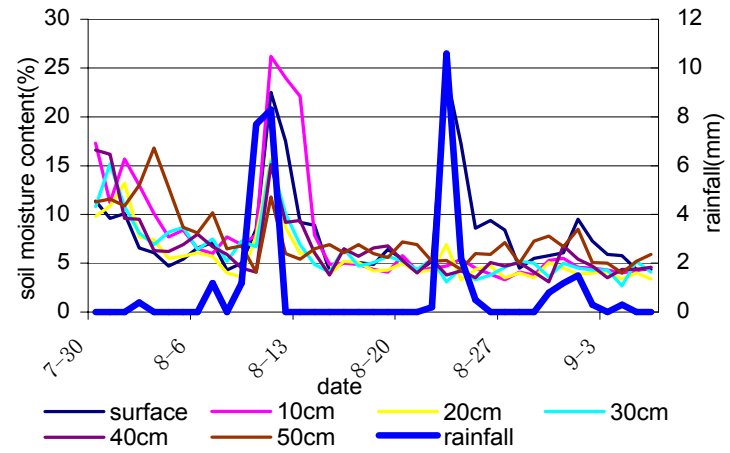

Figure 5: Observation data in the third observation point.

\section{Data analysis and EAR determination}

\subsection{Observation data analysis}

The data illustrated in figure 3-5 indicate that the soil moisture content increased rapidly on the day with rainfall and decreased rapidly after the rainfall and kept a relatively stable value about one week later. The same tendency of soil moisture content decay appears in different observation points with different conditions and different depth of these profiles. In these observation points, the conditions 
of the first observation point are most similar to the source region of debris flow in Jiangjia Gully where debris flow forms from steep slopes while the soil on the slope becomes unstable under the triggering of rainfall. Furthermore, debris flow forms from the shallow soil saturated by rainfall on the slope according to the research of Cui et al (2003) and the field observation. The data of the $20 \mathrm{~cm}$ depth of the first observation point are selected as the typical data to be analyzed.

According to the data analysis, following conclusions about the decay process of soil moisture content can be deduced:

(1) The soil moisture content has a relatively stable value although there is no rainfall in a long period. This value can be considered as the basic soil moisture content without relation to any specific rainfall event. So this value can be set to a constant $c$ not included in $E W_{i}$.

(2) $E W_{i}=W$ while $i=0$ and $E W_{\mathrm{i}}=0$ while $W=0$.

(3) $E W_{i}$ should be the monotonic decreasing function of $i$ and monotonic increasing function of $W$.

(4) $E W_{i}$ decreases very rapidly while $i$ increasing and should be convergent while it is summed from 0 to $\infty$.

Based on these conclusions, $E W_{i}=g(W, i)$ can be assumed as:

$$
E W_{i}=W \times \frac{i+a^{k}}{(i+a)^{k}}
$$

Where $a$ and $k$ are constants. Then the total soil moisture content $E W$ is:

$$
E W=E W_{1}+E W_{2}+\cdots+E W_{n}+c=\sum_{i=1}^{n} W \times \frac{i+a^{k}}{(i+a)^{k}}+c
$$

Because there is no rainfall for a long period before 10 August and from 11 August to 22 August, the process of soil moisture content decreasing can be considered independently. So the observation data from 10 to 22 August are selected to fit equation (4) with least-squares procedure. In the fitting, $k$ is set to 3,4 and 5 separately because $k$ must be more than 2 in order to make equation (4) convergent. The fitting results indicate 3 is best for $k$, and when $k=3, a=0.08$, $c=4.02$. Then

$$
E W_{i}=W \times \frac{i+0.08^{3}}{(i+0.08)^{3}}
$$

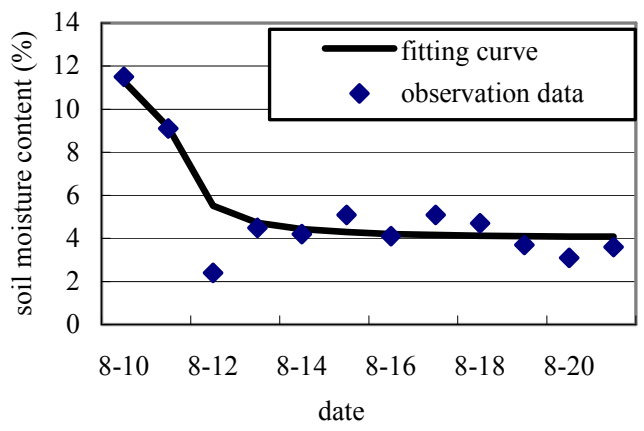

Figure 6: Comparison of fitting curve and observation data. 
The confidence interval of the fitting under $95 \%$ credence of $a$ and $c$ are $(-0.02746,0.1868)$ and $(3.565,4.479)$, and the error of mean square root is 0.618 . The fitting curve and the observation data are shown in figure 6.

\subsection{Determination of EAR}

According to the analysis on the relation between the decay processes of soil moisture content and $\mathrm{EAR}, E F_{i}=f(F, i)$ and $E W_{i}=g(W, i)$ have same functional form. According to equation (5), the effective rainfall of the rainfall event on the $i$-day before can be expressed as:

$$
E F_{i}=F \times \frac{i+0.08^{3}}{(i+0.08)^{3}}
$$

Under the assumption that the decrease of EAR of different rainfall events is mutually independent and according to equation (1), the total EAR is expressed as:

$$
E F=\sum_{i=1}^{n} F \times \frac{i+0.08^{3}}{(i+0.08)^{3}}
$$

Although equation (7) is convergent, in order to make the calculation simple, the effective rainfall of some rainfall events can be neglected while it decreases to less than $0.1 \mathrm{~mm}$. If ten such events are neglected, the neglected effective rainfall amount is only $1 \mathrm{~mm}$ and this value has almost no influence on the result of debris flow forecast which is set up based on statistics approach. The span of $n$ becomes limited under this simplification and the calculation is simplified in practice.

\section{Conclusions and discussion}

The following conclusions are drawn from the analysis of field observation data as well as the model of determining EAR.

(1) EAR impacts the initiation of debris flow and is an important factor for debris flow forecast.

(2) For soil-mechanics-typed debris flow, the EAR impacting debris flow initiation can be reflected in soil moisture content. And the soil moisture content and effective rainfall decay in the same manner after a rainfall event.

(3) Under the assumption that the decrease processes of effective rainfall from different rainfall events are mutually independent, the total EAR is the linear superimposition of the EAR of each rainfall event.

(4) According to the analysis on the observation data of rainfall and soil moisture content in Jiangjia Gully, Yunnan Province, the equation of calculating the antecedent effective rainfall for debris flow forecast is $E F=\sum_{i=1}^{n} F \times \frac{i+0.08^{3}}{(i+0.08)^{3}}$.

However, EAR decays in different forms and brings different impacts on debris flow initiation depending upon environment conditions. The following problems should be further discussed.

(1) For soil-mechanics-typed debris flow, EAR impacts debris flow initiation through changing the soil moisture content, and for hydromechanics-typed debris 
flow, it impacts debris flow initiation through changing the runoff. However, only the former is discussed in this paper. The latter should be discussed in later research, and a more general formula for both types of debris flows is needed to supply a simple approach of determining EAR for debris flow forecast.

(2) Regional difference is important for determining EAR because the decay process of soil moisture content is influenced by many factors such as climate, vegetation, soil, geomorphology etc. However, study in this paper is carried out based on observations in a given catchment, Jiangjia Gully, the application of the EAR formula must be limited to regions with similar natural conditions. In order to satisfy the requirement of debris flow forecast in different regions, formulas for different regions have to be set up by more observations in later research.

\section{Acknowledgement}

This research is supported by the Knowledge Innovation Program of Chinese Academy of Sciences (KZCX3-SW-352).

\section{References}

[1] Senoo, K. et al. Rainfall indexes for debris flow warning evacuating program. Shin-Sabo, 1985, 38(2):16-21. (in Japanese)

[2] Kubota, T. et al. A study on a Neural Network system for critical rainfall determination of debris flow warning and Evacuation. Journal of the Japan Society of Erosion Control Engineering, 1995, 47(6): 8-14. (in Japanese)

[3] Fujii, K. et al. Study on the Accuracy of warning and evacuation timing for debris flow. Journal of the Japan Society of Erosion Control Engineering, 1994, 47(2): 35-42. (in Japanese)

[4] Hayashi, T. et al. Nobutomo Osanai. Study on the management of critical rainfall determination of debris flow warning. Journal of the Japan Society of Erosion Control Engineering, 2000, 53(2): 57-61. (in Japanese)

[5] Senoo, K. et al. On the theme and improvement of standard rainfall of warning and evacuation from sediment disasters. Journal of the Japan Society of Erosion Control Engineering, 2001, 53(6): 37-44. (in Japanese)

[6] Tan, W. The character of rainfall vertical distribution and critical rainfall of debris flow forecast in Babuli Gully. Sichuan Meteorology (in Chinese), 1988, 8(2): 25-28.

[7] Tan, W. Distribution characters of critical rainfall line for the debris flow gully. Bulletin of Water and Soil Conservation, 1989, 9(6): 21-26. (in Chinese)

[8] Tan, B. et al. Study on prediction for rainstorm debris flow along mountain district railways. Journal of Natural Disasters, 1995, 4(2): 43-52. (in Chinese)

[9] Chen, J. Study on debris flow forecast in Jiangjia Gully. In: Observation and Research on Debris Flows in Jiangjia Gully in Yunnan(ed. Wu, J., Kang, Z. et al), Beijing: Science Press, 1990, 197-213. (in Chinese) 
22 Monitoring, Simulation, Prevention and Remediation of Dense Debris Flows II

[10] Fan, J. C. et al. Determination of critical thresholds for debris-flow occurrence in central Taiwan and their revision after the 1999 Chi-Chi great earthquake. In: Debris Flow Hazard Mitigation: Mechanics, Prediction, and Assessment (ed. Rickenmann, D. and Chen, C. L.), Proceedings 3rd International DFHM Conference, Davos, Switzerland, September 10-12, Rotterdam: Millpress, 2003, 103-114.

[11] Fedora, M. A. et al. Storm runoff simulation using an antecedent precipitation index(API) model . Journal of hydrology, 1989, 112:121-133.

[12] Wu, J. et al. Debris flow and its prevention (in Chinese). Beijing: Science Press, 1993, 81-89.

[13] Cui, P. et al. Relationship between occurrence of debris flow and antecedent precipitation: taking the Jiangjia Gulley as an Example. Science of Soil and Water Conservation, 2003, 1(1): 11-15. (in Chinese) 